\title{
Bladder-Sparing Approach with Radiotherapy in Patients with Small Cell Carcinoma of the Bladder
}

\author{
Hiroko Akamatsu1 ${ }^{*}$, Takuma Nomiya, ${ }^{1,2}$, Mayumi Harada1,2, Ibuki Oota, ${ }^{1,3}$, \\ Mayumi Ichikawa1, Misako Miwa1, Yuuki Kuroda', Kenji Takai', Akira Anbai' \\ Hideo Kawaguchi, Yoshihiro Takai, ${ }^{6}$ Kenji Nemoto' ${ }^{1}$ \\ ${ }^{1}$ Department of Radiation Oncology, School of Medicine, Yamagata University, Yamagata, Japan \\ ${ }^{2}$ Research Center Hospital for Charged Particle Therapy, National Institute of Radiological Sciences, \\ Chiba, Japan \\ ${ }^{3}$ Department of Radiation Oncology, Iwate Prefectural Central Hospital, Morioka, Japan \\ ${ }^{4}$ Department of Radiation Oncology, Yamagata City Hospital Saiseikan, Yamagata, Japan \\ ${ }^{5}$ Department of Radiation Oncology, School of Medicine, Akita University, Akita, Japan \\ ${ }^{6}$ Department of Radiology, School of Medicine, Hirosaki University, Hirosaki, Japan \\ Email: ${ }^{\text {h.akamatsu@med.id.yamagata-u.ac.jp }}$
}

Received 7 May 2014; revised 5 June 2014; accepted 2 July 2014

Copyright (C) 2014 by authors and Scientific Research Publishing Inc.

This work is licensed under the Creative Commons Attribution International License (CC BY).

http://creativecommons.org/licenses/by/4.0/

cC) (i) Open Access

\section{Abstract}

Small cell carcinoma (SCCB) of the bladder is rare and has a poor prognosis. Because of its rarity, gold standard care has not been established. The purpose of this study was to analyze the feasibility of a bladder-sparing approach. Data for treatments and outcomes in patients with a diagnosis of SCCB who received bladder-sparing treatment in our facility in the period from February 2007 to August 2012 were retrospectively analyzed. Six eligible patients received definitive radiotherapy in the cancer network of Tohoku region. Mean age of the patients was 69.5 years (range: 44 83 years), and median follow-up period was 29.2 months (range: 4.1 - 54.5 months). The mean dose was $60 \mathrm{~Gy}$ (range: 60 to $63 \mathrm{~Gy}$ ), and 1.2 - 2.0 Gy was given per fraction. The initial CTV was the whole pelvis or the small pelvis, and it was shrunk to the bladder or tumor as a boost. The 1-year and 3 -year overall survival rates were $83.3 \%$ and $55.6 \%$, respectively. The 1 -year and 3-year local control rates were both $\mathbf{8 0 . 0 \%}$. Only one patient had local failure. Recurrence or metastasis in sites other than the brain occurred in three $(50 \%)$ of the patients after primary treatment. Two of those patients did not receive any chemotherapy. None of the patients had serious toxicities, and the bladder could be preserved in all patients. In this study, radiotherapy was effective for patients with SCCB. There was no recurrence in the bladder, and it was possible to preserve the

${ }^{*}$ Corresponding author.

How to cite this paper: Akamatsu, H., Nomiya, T., Harada, M., Oota, I., Ichikawa, M., Miwa, M., Kuroda, Y., Takai, K., Anbai, A., Kawaguchi, H., Takai, Y. and Nemoto, K. (2014) Bladder-Sparing Approach with Radiotherapy in Patients with Small Cell Carcinoma of the Bladder. Journal of Cancer Therapy, 5, 797-805. http://dx.doi.org/10.4236/jct.2014.58087 
bladder in all patients. Distant metastases were observed frequently, and chemotherapy was considered to be essential. Local treatment with radiotherapy has an important role from the point of view of the patient's QOL.

\section{Keywords}

Small Cell Carcinoma of the Bladder, Radiotherapy, Cystectomy

\section{Introduction}

A case of small cell carcinoma of the bladder (SCCB) was first reported in 1981 [1]. Small cell carcinoma of the bladder is a rare disease. The incidence of SCCB has been reported to be 0.05 to 0.14 cases per 100,000 population, accounting for only $0.3 \%$ to $0.6 \%$ of all bladder cancers [2]. Data for 533 patients were deposited in the Surveillance, Epidemiology, and End Results (SEER) Medicare database from 1991 to 2005 in the United States [3]. The ratio of men to women was reported to be 3:1 [2]. The SEER database shows that the incidence of SCCB in the United States has been gradually increasing and the incidence is expected to continue increasing in the future [2].

Cases of small cell carcinoma have been documented in multiple sites of origin, most commonly the lung (13\% - 20\%) [4] [5]. The common sites of origin of extrapulmonary small cell carcinoma are the oesophagus $(0.8 \%-2.4 \%)$, larynx $(0.5 \%-1.0 \%)$, and bladder $(0.3 \%-1.0 \%)$ [4]. Small cell carcinoma is a distinct clinical and histological entity within the cancer of each organ because of its aggressive clinical behavior [4]. The clinical features of small cell lung carcinoma (SCLC) are a tendency for early dissemination, high initial response rate for chemotherapy, and high frequency of metastases [5].

Although a gold standard for treatment of SCCB has not been established, the most common procedure as definitive treatment is surgery [6]. Patients who undergo cystectomy must also undergo urostomy, which imposes a burden on their daily life. Recently, a multidisciplinary approach including radiotherapy has shown promise for local treatment of SCCB [7]. The purpose of this study was to analyze the feasibility of a bladder-sparing approach with radiation therapy.

\section{Methods}

\subsection{Patients}

The database of patients who had received radiotherapy during the period from February 2007 to August 2012 was investigated in the cancer network of Tohoku region. A search was made for patients in each facility using the keywords, "bladder carcinoma" and "small cell carcinoma". All of the patients were diagnosed histopathplogically on the basis of WHO criteria. Histological diagnosis was performed with a biopsy or a specimen that had been resected from the bladder tumor with transeurethral resection of the bladder tumor (TUR-BT). In analogy to small cell carcinoma of the lung, patients with any local stage, no distant metastases, and involvement of maximally one locoregional lymph node of less than $2 \mathrm{~cm}$ were determined to have limited diseases (LD), and all others were determined to have extensive diseases (ED). All patients gave written informed consent for the treatment.

\subsection{Radiotherapy}

Different approaches have been used of the facilities as a regimen of radiation therapy. In most cases, radiotherapy performed using $10 \mathrm{MV}$ photons with four beams as a box technique (Figure 1(a) and Figure 1(b)). The treatment was all planned on the basis of CT. The median dose was $60 \mathrm{~Gy}$ (range, 60 to $63 \mathrm{~Gy}$ ), and 1.2 - 2.0 Gy was given per fraction. Irradiation was performed once or twice a day and generally performed after urination. Patients were irradiated with a total dose of 60 - 63 Gy with conventional fractionation $(n=3)$ or hyperfractionation (HF) $(n=3)$. The clinical target volume (CTV) was the whole pelvis or a small part of the pelvis at first and was shrunk to the bladder or tumor as a boost (Figure 1(c) and Figure 1(d)). The initial CTVs were the whole pelvis in four patients, a small part of the pelvis in one patient, and the bladder in one patient. Boost- 

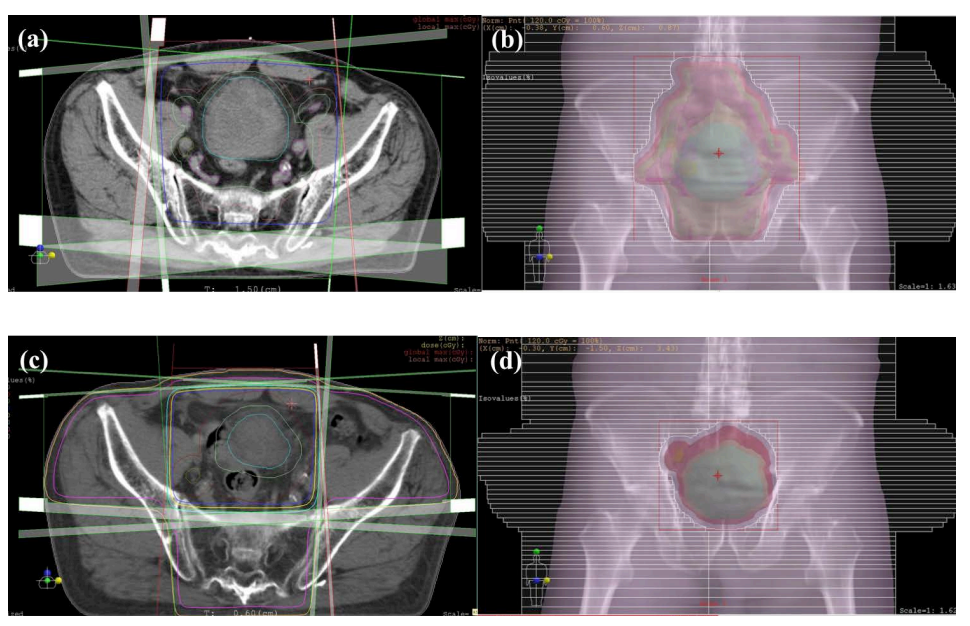

Figure 1. Dose distribution of the whole pelvis and bladder. This is the dose distribution of the three-dimensional radiotherapy plan for SCCB in our institution. At first, CTV was the whole pelvis, and irradiation $30 \mathrm{~Gy}$ with in irradiation 25 fractions was performed. CTV was then shrinked to the bladder and tumor, and irradiation with $30 \mathrm{~Gy}$ in 25 fractions was performed. Total dose was $60 \mathrm{~Gy}$ in 50 fractions (2 fractions per day).

CTVs were the bladder and tumor in four patients. In two patients, there was no shrinkage in the initial CTV of few completion of radiotherapy. In one patient, the whole pelvis was irradiated with a total dose of $63 \mathrm{~Gy}$ in 35 fractions. Another patient was irradiated to only pelvis with a total dose of $60 \mathrm{~Gy}$ in 50 fractions (HF). Prophylactic cranial irradiation was not performed.

\subsection{Chemotherapy}

Chemotherapy was performed preceding or concomitantly with radiotherapy. The chemotherapy regimens used were platinum-based regimens that are known to have activity against small cell carcinoma of the lung. A multidrug regimen combined with cisplatin (CDDP) and with etoposide and irinotecan was mostly used. For elderly patients, carboplatin (CBDCA) was used instead of CDDP $(n=1)$. Depending on the general condition of the patient, 2 to 4 courses of chemotherapy were given. Chemotherapy was given concomitantly with radiotherapy in most cases but was given before radiotherapy in some cases.

\subsection{Analysis}

Overall survival, progression-free survival, and local control were determined from the date of starting chemotherapy or radiotherapy to the date of confirmation of events, and the Kaplan-Meyer method was used to calculate cumulative rates. Local control rate and progression-free survival rate were also calculated. Early toxicity was evaluated retrospectively on the basis of the Common Terminology Criteria for Adverse Events ver.4.0 (CTCAE v4.0) from medical redords. All cases were evaluated for the existence and severity with RTOG/ EORTC scales.

\section{Results}

Eleven patients with SCCB were identified from our institutions. Six of these patients had received bladdersparing radiotherapy, and data for these patients were retrospectively analyzed. Five patients who had received cystectomy before radiotherapy were excluded. The excluded patients had received palliative radiotherapy for brain metastasis or recurrent tumor. The general status of each patient was good with performance state (PS) from 1 to 2 by the WHO score. Only one patient after cerebral infarction was PS 2 (Table 1, Pt. 6). Table 1 shows the patient characteristics including clinical stage and treatment. The median follow-up period was 29.2 months (range: 4.1 - 54.5 months). All patients were male, and the mean age was 69.5 years (range: 44 - 83 years). Details of radiotherapy and chemotherapy are summarized in Table 1. 
Table 1. Patient, tumor and treatment characteristics.

\begin{tabular}{|c|c|c|c|c|c|c|c|c|c|c|c|}
\hline Pt. & Age & Sex & $\begin{array}{l}\text { Pathology } \\
\text { findings }\end{array}$ & Stage & Radiotherapy & Dose & $\mathrm{Cx}$ & Cx start & Relapse site & $\begin{array}{l}\text { Follow-up } \\
\text { (month) }\end{array}$ & Status \\
\hline 1 & 62 & M & mixed & ED & WP & 60 Gy/50fr. (HF) & $\mathrm{P} / \mathrm{E} / \mathrm{I}$ & Concurrent & - & 48.9 & NED \\
\hline 2 & 44 & M & mixed & LD & SP + Bladder & 60 Gy/30fr. & $\mathrm{P} / \mathrm{E} / \mathrm{I}$ & Before RT & Brain & 33.0 & DOD \\
\hline 3 & 71 & M & Pure & ED & WP + Bladder & 63 Gy/35fr. & $\mathrm{P} / \mathrm{E}$ & Concurrent & - & 54.5 & NED \\
\hline 4 & 68 & M & Pure & ED & WP + Bladder & 60 Gy/50fr. (HF) & $\mathrm{C} / \mathrm{E}$ & Before RT & Liver, Plura, Brain & 11.8 & AWD \\
\hline 5 & 83 & M & Pure & LD & Bladder & 60 Gy/30fr. & - & - & $\begin{array}{l}\text { Pelvis, PALN, } \\
\text { Lung }\end{array}$ & 25.4 & AWD \\
\hline 6 & 77 & M & pure & LD & WP + Bladder & 60 Gy/50fr. (HF) & - & - & PALN & 4.1 & DOA \\
\hline
\end{tabular}

Abbreviations: $\mathrm{RT}=$ radiation, $\mathrm{LD}=$ limited disease: $\mathrm{TXN0}-1 \mathrm{M} 0, \mathrm{ED}=$ extensive disease: $\mathrm{TXN2}-3 \mathrm{M0}-1$, WP = whole pelvis, $\mathrm{SP}=$ small pelvis, CR = complete response, $\mathrm{HF}=$ hyperfractionation, $\mathrm{Cx}=$ chemotherapy, $\mathrm{P}=$ cisplatin, $\mathrm{E}=$ etoposide, $\mathrm{C}=$ carboplatin, $\mathrm{I}=$ irinotecan, $\mathrm{PALN}=$ para-aortic lymphnodes, OS = overall survival, $\mathrm{CR}=$ complete response, $\mathrm{NED}=$ no evidence of disease, $\mathrm{AWD}=$ alive with disease, $\mathrm{DOD}=$ dead of disease, DOA = dead of another disease.

The 1-year and 3-year overall survival rates were 83.3\% and 55.6\%, respectively. The 1-year and 3-year local control rates were both $80.0 \%$. The median progression-free survival period was 11.2 months (range: 3.6 - 54.5 months, 95\% confidence interval (CI): 0 - 24.6). Only one patient had local failure in the follow-up period (Figure 2). Treatments and outcomes are summarized in Table 1. All patients responded well and achieved complete remission after radiotherapy, but one of the six patients had local failure. One patient with hematuria at the onset developed difficulty in urination, and bilateral nephrostomy catheters were inserted before treatment (Pt. 4). After the initial treatment, the nephrostomy catheters were removed. However, the bladder was preserved without urinary diversion in all patients during the observation period.

Recurrence or metastasis in sites other than the brain occurred in three (50\%) of the patients after primary treatment (Figure 3). Metastasis to the para-aortic lymph node region occurred in two of the six patients. Metastasis was also found in various sites including the liver and pleura.

Brain metastasis was found in two patients in the follow-up period (Table 1). Brain metastasis in one patient occurred 18 months after treatment, though local and systemic metastases in sites other than the brain were controlled.

Early adverse events are shown in Table 2, and late morbidity of the treatment is shown in Table 3. Gross hematuria was observed in five patients at diagnosis and disappeared after treatment. Microscopic hematuria was observed in five patients. Hematuria was continuously observed in one patient during observation period (Pt. 6) and was observed intermittently in the other patients. Except for blood hematotoxity, no early adverse reactions of more than grade 3 were observed (Table 2). There were no severe late adverse reactions in the observation period. Grade 1 of late adverse reaction in the bladder was observed in two patients (Pt. 3 and Pt. 4). Gross hematuria from a bladder diverticulum occurred in the one patient 26 months after initial treatment (Pt. 3). The gross hematuria disappeared after one week of conservative management. There was no microscopic or gross hematuria after the diverticular bleeding had stopped.

\section{Discussion}

According to the literature, the most frequently used definitive treatment method for SCCB seems to be surgery [2] [3] [6]. Koay et al. compared cystectomy with bladder-sparing approaches in a retrospective study [3]. The bladder-sparing approach involved transurethral resection of the bladder tumor combined with chemotherapy and radiation [3]. Their comparison showed that there was no difference in terms of overall survival or cancerspecific survival $(p<0.05$ ) [3]. On the other hand, it was shown that radiation improved overall survival in patients with regional-stage disease $(p<0.05)$ [3]. Our data also showed that radiotherapy was effective for controlling a tumor in the pelvis. An effect on symptoms of gross hematuria was also observed. In five patients with hematuria as the initial presentation, the gross hematuria disappeared after radiotherapy. Local control without severe complications such as bleeding and constriction of the bladder was accomplished in all patients. For the patient's quality of life, it is reasonable to consider radiotherapy as the first choice. Cheng et al. reported 64 patients with small cell carcinoma of the bladder [8]. Thirty-eight patients (59\%) underwent cystectomy and 66\% of the patients had lymph node metastases at the time of cystectomy [8]. The 1-year and 5-year disease-specific 


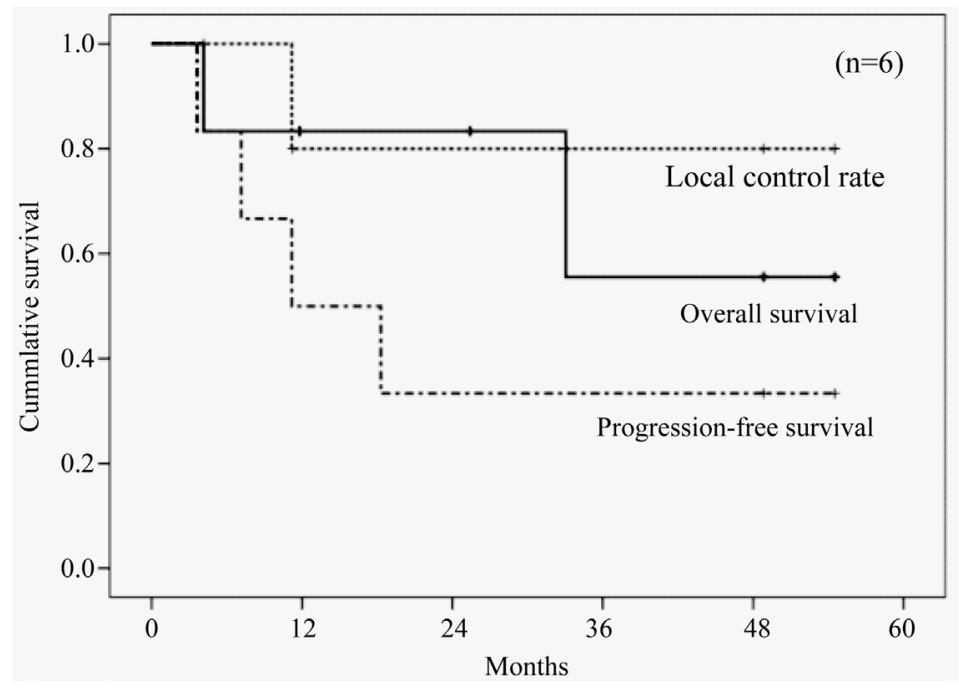

Figure 2. Local control, overall survival and progression-free survival in patients with SCCB. Kaplan-Meier survival curves for patients with SCCB show OS (solid line), local control rate (dashed line) and PFS (another dashed line).

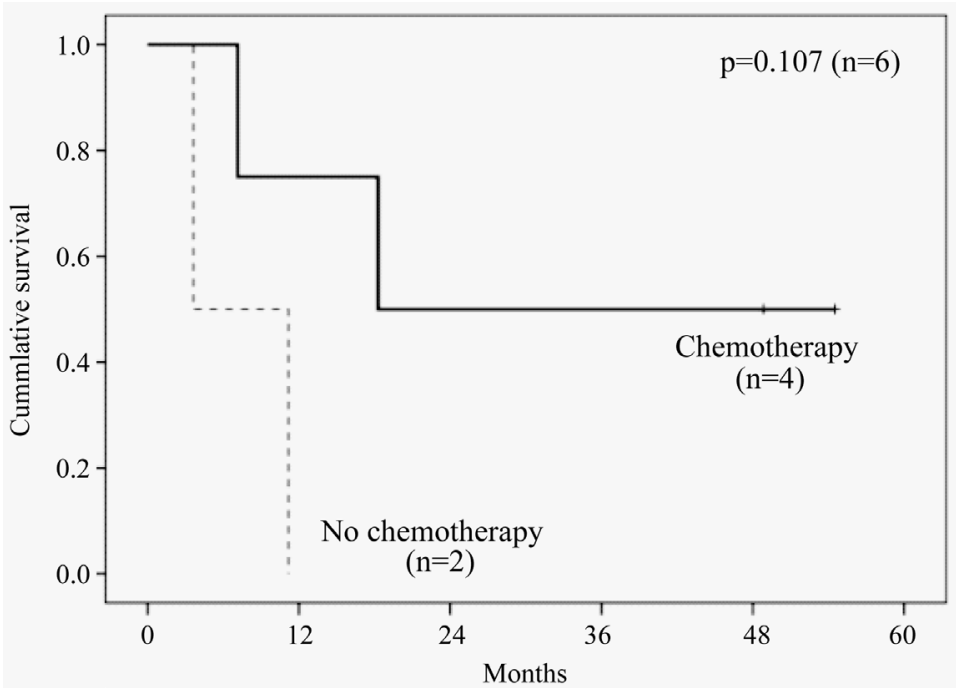

Figure 3. Progression-free survival of patients who received chemotherapy that of patients who did not receive chemotherapy. Kaplan-Meier survival curves are shown for patients with SCCB stratified according to treatment with chemotherapy (solid line) or without chemotherapy (dashed line). Two patients with no chemotherapy had local and distant relapse within 12 months after radiotherapy. No significant difference was found between the two groups $(\mathrm{p}=0.107)$.

survival rates in patients who underwent cystectomy were 57\% and 16\%, respectively, and 1-year and 5-year disease-specific survival rates in patients who did not undergo cystectomy were 55\% and 18\%, respectively [8]. No significant survival difference was found between patients who underwent cystectomy and those who did not $(\mathrm{p}=0.65)[8]$.

Schreiber et al. reported overall survival for 109 patients with stage II disease [6]. The median OS of the patients who underwent cystectomy was 40 months (95\% CI: 5.5 - 74.5), and the 3-year and 5-year OS rates for those patients were $51.7 \%$ and $48.7 \%$, respectively [6]. On the other hand, the median OS of patients who were treated with radiotherapy alone was 18 months (95\% CI: 16.7 - 35.3), and the 3-year and 5-year OS rates for 
Table 2. Acute toxicity according to the bladder-sparing approach in the patients with the SCCB.

\begin{tabular}{ccccc}
\hline & Grade 0 & Grade 1 & Grade 2 & Grade $>3$ \\
\hline WBC & 1 & 0 & 1 & 4 \\
Hb & 0 & 2 & 2 & 2 \\
Plat & 2 & 1 & 2 & 0 \\
Hematuria & 1 & 5 & 0 & 0 \\
Obstruction & 4 & 0 & 2 & 0 \\
Diarrhea & 6 & 0 & 0 & 0 \\
\hline
\end{tabular}

Abbreviations: $\mathrm{WBC}=$ white blood cells, $\mathrm{Hb}=$ hemoglobin, Plat $=$ platelet.

\section{Table 3. RTOG/EORTC late radiation morbidity scoring scheme.}

\begin{tabular}{|c|c|c|}
\hline & Bladder & Number of patients \\
\hline Grade 1 & Slight epithelial atrophy; minor telangiectasia (microscopic hematuria) & 0 \\
\hline Grade 2 & Moderrate frequency; generalized telangiectasia; intermittent macroscopic hematuria & 0 \\
\hline Grade 3 & Obstruction or bleeding requiring surgery & 0 \\
\hline \multirow[t]{2}{*}{ Grade 4} & Necrosis / perforation fistula & 0 \\
\hline & Rectum & Number of patients \\
\hline Grade 1 & Mild diarrhea; mild cramping: bowel movement 5 times daily; slight discharge or bleeding & 0 \\
\hline Grade 2 & $\begin{array}{l}\text { Moderate diarrhea and colic; bowel movement > } 5 \text { times daily; excessive rectal mucus; } \\
\text { intermittent bleeding }\end{array}$ & 0 \\
\hline Grade 3 & Obstruction or bleeding requiring surgery & 0 \\
\hline Grade 4 & Necrosis/perforation fistula & 0 \\
\hline
\end{tabular}

those patients were $30.1 \%$ and $25.1 \%$, respectively [6]. OS was significantly better for patients who underwent cystectomy $(p=0.01)$ [6]. Since the study by Schreiber et al. was a retrospective study using a large database, detailed information about the patients in each group was not sufficient. For patients with comorbidities or of advanced age for whom surgery or chemotherapy is difficult, radiotherapy might be considered as a potential adaptation. There is a possibility of some selection bias with regard to which patients received cystectomy or definitive radiation.

Local control was achieved in 5 of the 6 patients in the present study, and rate of local control in the literature is quite high. When considering QOL of the patients, cystectomy may not always be necessary for localized SCCB.

Karpman et al. reviewed 23 reported cases of SCCB treated with primary chemoradiotherapy [9]. In their series, the median radiation dose was 60 Gy [9]. Seventy percent of the patients were alive after a median followup period of 34 months, and local control was achieved in most of the patients [9]. In our facilities, the most frequently used radiation dose for SCCB was $60 \mathrm{~Gy}$. The HF technique was used at the discretion of each facility, because it seemed to be reasonable for preventing gastrointestinal toxicity. The final results of treatment outcome will need a longer follow-up period, but the initial therapeutic effect in the bladder was good, with minimal acute adverse effects.

In our study, only one of the six patients developed local recurrence in the field margin that was irradiated with approximately $30 \mathrm{~Gy}$, and there was no patient who developed local recurrence when the entire bladder was irradiated with more than $50 \mathrm{~Gy}$. The patient who developed local recurrence was an 83-years-old man (Table 1, Pt. 5). He was irradiated with 30 Gy to a small part of the pelvis, and irradiation was continued with a total dose of $60 \mathrm{~Gy}$ to his bladder and tumor. There was local recurrence in external iliac lymph node of the field margin, which was irradiated with approximately 30 Gy 11.8 months after radiotherapy. Salvage surgery was not performed, and chemotherapy was performed after the recurrence had been detected. He is alive with disease at 25.4 months after radiotherapy. From these data, 60 Gy to the GTV can achieve a good local control rate without late severe toxicity. Irradiated fields seem to be favorable for local control including the lymph node re- 
gion in the pelvis. Although the usefulness of prophylactic nodal irradiation remains unclear, there was lymph node recurrence in the para-aortic region in 2 of the 6 patients. Both of whom had a recurrence in PALN didn't received chemotherapy in initial treatment because of their advanced age and comorbidity. One patient was 77-year-old man with limited disease and slightly decreased respiratory function. Extensive local and systemic relapse occurred 11 months after treatment (Table 1, Pt. 5). The other patient was an 83-year-old man with limited disease (Table 1, Pt. 5). Local and distant relapse occurred in external iliac lymph node, PALN and lung 11.8 months after radiotherapy. There was no recurrence in his bladder. When patients who received cystectomy were included, metastasis to the para-aortic lymph nodes was observed in $45.5 \%$ of the patients $(n=5 / 11)$. However, most patients with PALN relapse also had systemic relapse at a same time. The trend for relapse has been equivocal so far. As mentioned above, further investigation of those hypotheses is needed because the small number of patients in the present study is an obvious limitation.

Mukesh et al. reported that the median survival period was 33 months for patients receiving chemotherapy vs 3 months for patients with no chemotherapy [10]. Bex et al. conducted a retrospective study on 17 patients with limited disease who were treated with chemo-radiotherapy at the Netherlands Cancer Institute [11]. All patients received platinum-based chemotherapy prior to local radiotherapy with 56 - 70 Gy [11]. A complete response was achieved in 15 patients (88\%) and median OS was 32.5 months $(p=0.028)$ [11]. The result of the present study is inconsistent with results of other studies [3] [10] [11]. Standard chemotherapy regimens for small cell carcinoma of the bladder have not been established. Systemic chemotherapy has an important role for small cell carcinoma of the bladder because the pathological characteristics of small cell carcinoma include a high tendency for early recurrence or metastasis. Patients with small cell lung cancer can show a high response rate to chemotherapy [5]. The first-line treatment of choice in extensive-stage SCLC is four to six cycles of etoposide combined with a platinum salt (cisplatin or carboplatin) [5]. For limited-stage SCLC, early administration of 45 Gy with concurrent etoposide and cisplatin at systemic doses is supported by current evidence [5].

As mentioned above, optimal regimens for extrapulmonary small cell carcinoma have not been clearly established [4]. However, many studies have shown that platinum-based regimens for small cell carcinoma of the lung were also effective for small cell carcinoma of extrapulmonary origins [4] [12]-[15].

Prophylactic cranial irradiation (PCI) for SCLC patients in complete remission after chemotherapy and radiotherapy has become a standard treatment. The incidence of brain metastasis ranges from $20 \%$ to $30 \%$ in SCLC patients with limited disease [16] and up to 40\% in SCLC patients with extensive disease [17]. Aupérin et al. reported that PCI reduced the risk of total brain metastasis (59\% vs. 33\% at 3 years) and improved survival rate (3-year survival from 15\% to 21\%) in SCLC patients [16]. Bex et al. reviewed reports on brain metastasis of small cell carcinoma of the bladder and reported that the pooled estimate of the cumulative incidence of brain metastasis was $10.5 \%$ (95\% CI: $7.5 \%$ to $14.1 \%)$ [18]. Although the incidence of brain metastases in SCCB was reported to be low compared with that in SCLC, brain metastasis occurred in two (33.3\%) of the six patients in this study. One patient had distant metastases in PALN at primary diagnosis, and multiple metastases occurred in the liver, plura, and brain (Pt. 4). The only site of recurrence in the other patient was the brain. Brain metastasis was found in that patient 18 months after primary treatment, and he had remained in complete remission in the extracerebral region for 33 months until he died (Pt. 2). Alrele et al. suggested that brain metastases were strongly asssosiated with the presence of bulky, higher stage tumors ( $\geq \mathrm{T} 3 \mathrm{~b}, \mathrm{~N}+\mathrm{or} \mathrm{M}+$ ) in SCCB [19]. It was reported that seven out of the eight patients with brain metastases had no progressive tumor in the extracerebral region [18]. When patients with ED achieve remission, they may benefit from PCI. If a multimodality approach including radiotherapy improves the prognosis of small cell carcinoma of the bladder, it is probable that brain metastasis will become a prognosis factor.

The most frequent early toxicity in this study was hematotoxity (Table 2). The patients with more than grade 3 of hematotoxity all received chemotherapy. Transurethral catheterization was needed in two patients (Table 2 and Table 3). In the patient with bladder tamponade that resulted from uncontrolled gross hematuria, bilateral nephrostomy catheters were inserted before treatment (Pt. 4). After the initial treatment, gross hematuria disappeared and the nephrostomy catheters were removed. However, a transurethral catheter was needed because of difficulty in urination. Another patient needed a transurethral catheter because of his general conditions as a result of pneumonitis (Pt. 6). Grade 1 of late adverse reaction in the bladder was observed in two patients (Pt. 3 and Pt. 4). Gross hematuria from bladder diverticulum occurred in one patient 26 months after initial treatment (Pt. 3). The gross hematuria disappeared after conservative management for a week. There was no microscopic or gross hematuria after the diverticular bleeding had stopped. 
The obvious limitations of this small retrospective study include a small number of patients, retrospective data, selection bias, and heterogeneous treatment. Further investigation and large-prospective evaluation to develop more effective therapies for SCCB are needed. However, this study showed the usefulness of bladder preservation in terms of patient's QOL. From such a point of view, there is an important role of this study as a preliminary study for further studies in the future.

\section{Conclusion}

Bladder-sparing therapy using chemotherapy and radiotherapy can result in good local control and may become one of the standard treatment choices.

\section{Acknowledgements}

The authors are grateful to Hirofumi Oikawa and Hisanori Ariga for their cooperation in the collection of the data.

\section{References}

[1] Cramer, S.F., Aikawa, M., Cebelin, M. and Cebelin, M. (1981) Neurosecretory Granules in Small Cell Invasive Carcinoma of the Urinary Bladder. Cancer, 47, 724-730. http://dx.doi.org/10.1002/1097-0142(19810215)47:4<724::AID-CNCR2820470417>3.0.CO;2-2

[2] Koay, E.J., Teh, B.S., Paulino, A.C. and Butler, E.B. (2011) A Surveillance, Epidemiology, and End Results Analysis of Small Cell Carcinoma of the Bladder: Epidemiology, Prognostic Variables, and Treatment Trends. Cancer, 117, 5325-5333. http://dx.doi.org/10.1002/cncr.26197

[3] Koay, E.J., Teh, B.S., Paulino, A.C. and Butler, E.B. (2012) Treatment Trends and Outcomes of Small-Cell Carcinoma of the Bladder. International Journal of Radiation Oncology * Biology * Physics, 83, 64-70. http://dx.doi.org/10.1016/j.ijrobp.2011.05.039

[4] Walenkamp, A.M., Sonke, G.S. and Sleijfer, D.T. (2009) Clinical and Therapeutic Aspects of Extrapulmonary Small Cell Carcinoma. Cancer Treatment Reviews, 35, 228-236. http://dx.doi.org/10.1016/j.ctrv.2008.10.007

[5] van Meerbeeck, J.P., Fennell, D.A. and De Ruysscher, D.K. (2011) Small-Cell Lung Cancer. Lancet, 378, $1741-1755$. http://dx.doi.org/10.1016/S0140-6736(11)60165-7

[6] Schreiber, D., Rineer, J., Weiss, J., Leaf, A., Karanikolas, N., Rotman, M. and Schwartz, D. (2013) Characterization and Outcomes of Small Cell Carcinoma of the Bladder Using the Surveillance, Epidemiology, and End Results Database. American Journal of Clinical Oncology, 36, 126-131. http://dx.doi.org/10.1097/COC.0b013e3182438c71

[7] Macedo, L.T., Ribeiro, J., Curigliano, G., Fumagalli, L., Locatelli, M., Carvalheira, J.B., Quintela, A., Bertelli, S. and De Cobelli, O. (2011) Multidisciplinary Approach in the Treatment of Patients with Small Cell Bladder Carcinoma. European Journal of Surgical Oncology, 37, 558-562. http://dx.doi.org/10.1016/j.ejso.2011.04.005

[8] Cheng, L., Pan, C.X., Yang, X.J., Lopez-Beltran, A., MacLennan, G.T., Lin, H., Kuzel, T.M., Papavero, V., Tretiakova, M., Nigro, K., et al. (2004) Small Cell Carcinoma of the Urinary Bladder: A Clinicopathologic Analysis of 64 Patients. Cancer, 101, 957-962. http://dx.doi.org/10.1002/cncr.20456

[9] Karpman, E., Goldberg, Z., Saffarian, A., Gandour-Edwards, R., Ellison, L.M. and de Vere White, R.W. (2004) Analysis of Treatment for Small Cell Cancer of the Bladder and Report of Three Cases. Urology, 64, 494-498. http://dx.doi.org/10.1016/j.urology.2004.04.038

[10] Mukesh, M., Cook, N., Hollingdale, A.E., Ainsworth, N.L. and Russell, S.G. (2009) Small Cell Carcinoma of the Urinary Bladder: A 15-Year Retrospective Review of Treatment and Survival in the Anglian Cancer Network. BJU International, 103, 747-752. http://dx.doi.org/10.1111/j.1464-410X.2008.08241.X

[11] Bex, A., Nieuwenhuijzen, J.A., Kerst, M., Pos, F., van Boven, H., Meinhardt, W. and Horenblas, S. (2005) Small Cell Carcinoma of Bladder: A Single-Center Prospective Study of 25 Cases Treated in Analogy to Small Cell Lung Cancer. Urology, 65, 295-299. http://dx.doi.org/10.1016/j.urology.2004.09.049

[12] Brenner, B., Tang, L.H., Shia, J., Klimstra, D.S. and Kelsen, D.P. (2007) Small Cell Carcinomas of the Gastrointestinal Tract: Clinicopathological Features and Treatment Approach. Seminars in Oncology, 34, 43-50. http://dx.doi.org/10.1053/j.seminoncol.2006.10.022

[13] Meng, M.B., Zaorsky, N.G., Jiang, C., Tian, L.J., Wang, H.H., Liu, C.L., Wang, J., Tao, Z., Sun, Y., Pang, Q.S., et al. (2013) Radiotherapy and Chemotherapy Are Associated with Improved Outcomes over Surgery and Chemotherapy in the Management of Limited-Stage Small Cell Esophageal Carcinoma. Radiotherapy \& Oncology, 106, 317-322. http://dx.doi.org/10.1016/j.radonc.2013.01.008 
[14] Renner, G. (2007) Small Cell Carcinoma of the Head and Neck: A Review. Seminars in Oncology, 34, 3-14. http://dx.doi.org/10.1053/j.seminoncol.2006.10.024

[15] Hatoum, G.F., Patton, B., Takita, C., Abdel-Wahab, M., La Fave, K., Weed, D. and Reis, I.M. (2009) Small Cell Carcinoma of the Head and Neck: The University of Miami Experience. International Journal of Radiation Oncology * Biology * Physics, 74, 477-481. http://dx.doi.org/10.1016/j.ijrobp.2008.08.014

[16] Auperin, A., Arriagada, R., Pignon, J.P., Le Pechoux, C., Gregor, A., Stephens, R.J., Kristjansen, P.E., Johnson, B.E., Ueoka, H., Wagner, H. and Aisner, J. (1999) Prophylactic Cranial Irradiation for Patients with Small-Cell Lung Cancer in Complete Remission. Prophylactic Cranial Irradiation Overview Collaborative Group. New England Journal of Medicine, 341, 476-484. http://dx.doi.org/10.1056/NEJM199908123410703

[17] Slotman, B., Faivre-Finn, C., Kramer, G., Rankin, E., Snee, M., Hatton, M., Postmus, P., Collette, L., Musat, E. and Senan, S. (2007) Prophylactic Cranial Irradiation in Extensive Small-Cell Lung Cancer. New England Journal of Medicine, 357, 664-672. http://dx.doi.org/10.1056/NEJMoa071780

[18] Bex, A., Sonke, G.S., Pos, F.J., Brandsma, D., Kerst, J.M. and Horenblas, S. (2010) Symptomatic Brain Metastases from Small-Cell Carcinoma of the Urinary Bladder: The Netherlands Cancer Institute Experience and Literature Review. Annals of Oncology, 21, 2240-2245. http://dx.doi.org/10.1093/annonc/mdq225

[19] Siefker-Radtke, A.O., Kamat, A.M., Grossman, H.B., Williams, D.L., Qiao, W., Thall, P.F., Dinney, C.P. and Millikan, R.E. (2009) Phase II Clinical Trial of Neoadjuvant Alternating Doublet Chemotherapy with Ifosfamide/Doxorubicin and Etoposide. Journal of Clinical Oncology, 27, 2592-2597. 
Scientific Research Publishing (SCIRP) is one of the largest Open Access journal publishers. It is currently publishing more than 200 open access, online, peer-reviewed journals covering a wide range of academic disciplines. SCIRP serves the worldwide academic communities and contributes to the progress and application of science with its publication.

Other selected journals from SCIRP are listed as below. Submit your manuscript to us via either submit@scirp.org or Online Submission Portal.
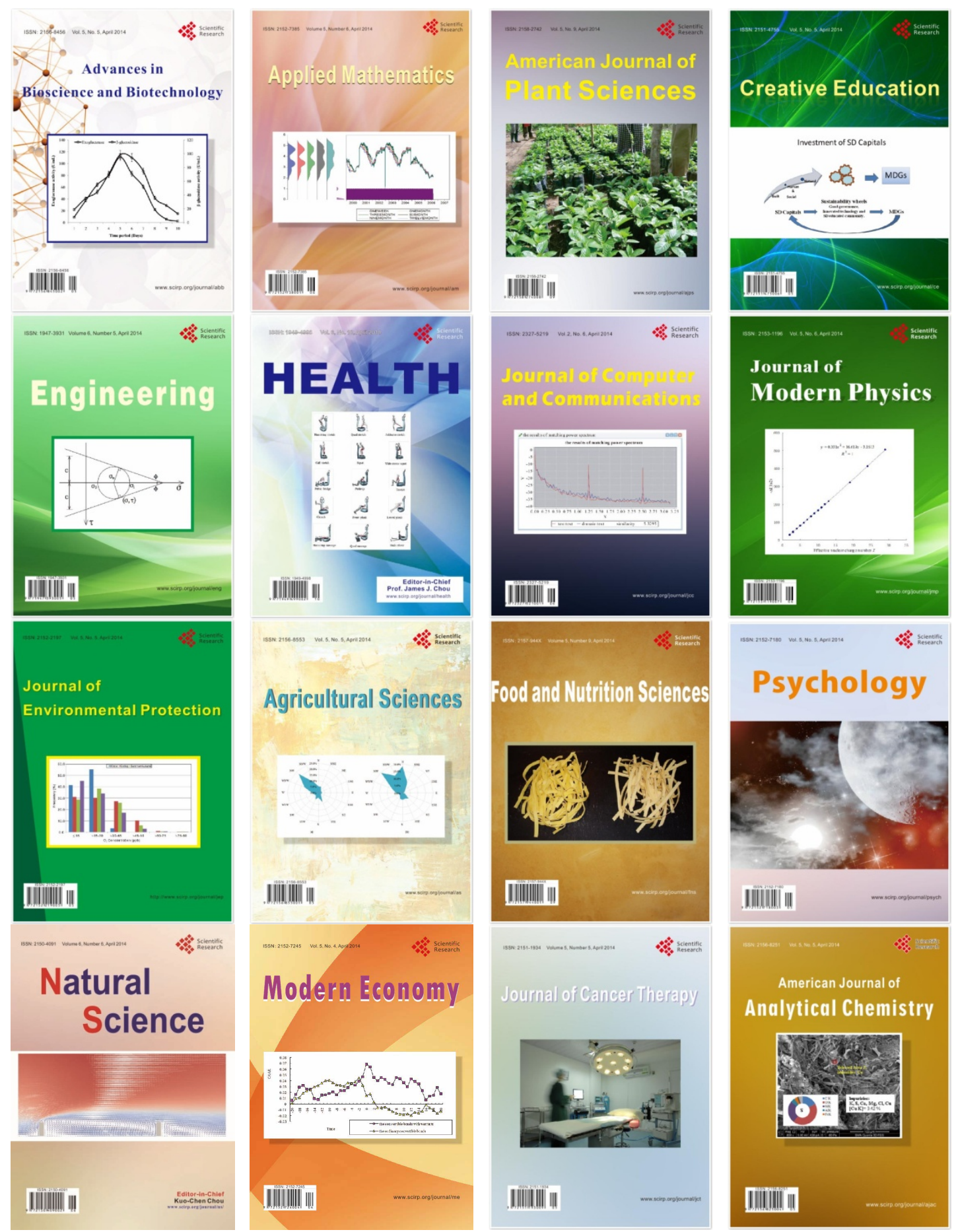